Quantitative analysis of the guidance of vortices in superconducting films with magnetic dots

M. P. González, E. Hollmann, and R. Wördenweber

Citation: Journal of Applied Physics 102, 063904 (2007);

View online: https://doi.org/10.1063/1.2781515

View Table of Contents: http://aip.scitation.org/toc/jap/102/6

Published by the American Institute of Physics

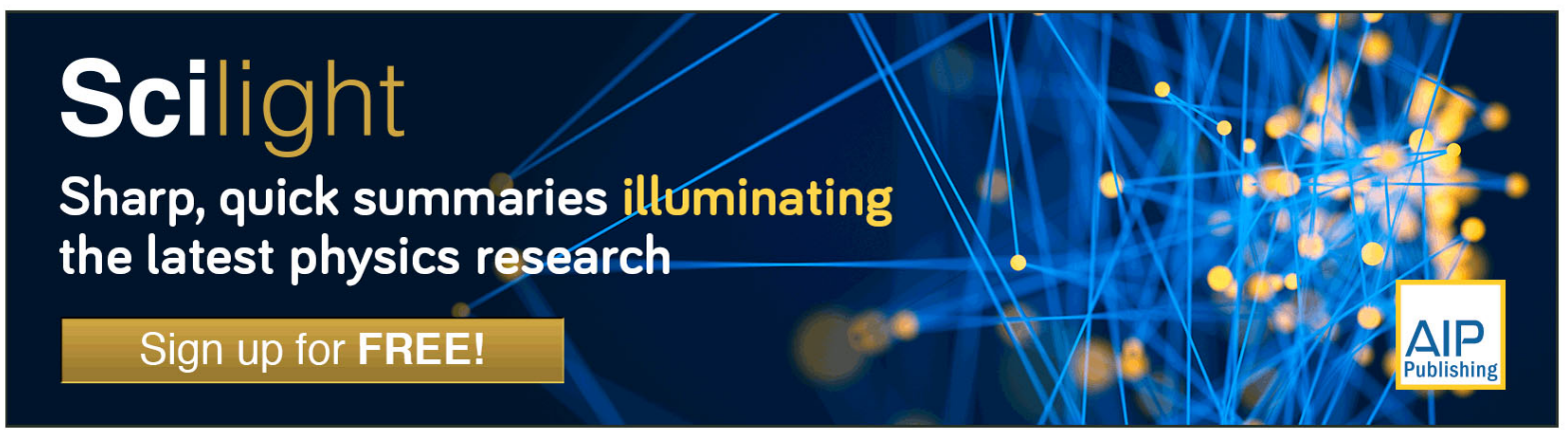




\title{
Quantitative analysis of the guidance of vortices in superconducting films with magnetic dots
}

\author{
M. P. González ${ }^{a)}$ \\ Departamento Física de Materiales, Facultad de Ciencias Físicas, Universidad Complutense de Madrid, \\ 28040 Madrid, Spain \\ E. Hollmann and R. Wördenweber \\ Institut für Bio-und Nanosysteme (IBN) und cni-Center of Nanoelectronic Systems for Information \\ Technology, Forschungszentrum Jülich, D-52425 Jülich, Germany
}

(Received 9 May 2007; accepted 29 July 2007; published online 19 September 2007)

\begin{abstract}
The guidance of vortices in superconducting $\mathrm{Nb}$ films with periodic arrangements of magnetic $\mathrm{Ni}$ dots is studied via simultaneous four-probe measurements in standard and Hall configuration. Ni dots are fabricated via $e$-beam lithography and lift-off technology. Arrays with periodicity down to $400 \mathrm{~nm}$ and dot size of $200 \mathrm{~nm}$ are obtained. Subsequently, the arrays are covered with a thin superconducting $\mathrm{Nb}$ layer. Simultaneous Hall and standard resistive measurements of the flux dynamic in $\mathrm{Nb}$ bridges with and without magnetic dots demonstrate and quantify the guidance of flux via the magnetic dots. Guidance of vortices is only possible in a limited current regime; guidance improves with decreasing temperature. Within the identified current regime most of the vortices ( $>40 \%$ for zero field) and in the optimum case all of the vortices are guided by the magnetic dots. () 2007 American Institute of Physics. [DOI: 10.1063/1.2781515]
\end{abstract}

\section{INTRODUCTION}

Vortices in type-II superconductors exhibit a large variety of interesting phenomena. The mutual interaction of vortices and the vortex-pin interaction gives rise to a rich variety of static and dynamic phases. Nowadays nanolithography techniques allow the fabrication of arrays of pinning centers with size, shape, and position controlled down to the submicrometric range. The resulting possibility to manipulate the properties of superconducting thin films on the nanoscale by adding nanostructures of nonsuperconducting materials has turned out to be of interest not only for basic research of superconductor behavior and vortex matter, but could also lead to applications of fluxonic devices.

Previously, the impact of magnetic nanostructures upon the properties of underlying superconducting films, especially the attractive interaction between magnetic dots and vortices, has been demonstrated. ${ }^{1-3}$ It has been shown that superconductivity can be induced by magnetic layers for nonzero magnetic fields, and that vortex pinning can be achieved by regular arrays of magnetic dots. The main goal of this work is to study the vortex dynamics and quantify the vortex guidance in superconducting thin films equipped with rows of magnetic nanodots. Experimentally this is achieved via a combination of simultaneous four-probe resistive measurements of the longitudinal and transversal (Hall) voltage in superconducting striplines with and without magnetic dots.

\section{EXPERIMENT}

The fabrication of small magnetic structures (e.g., magnetic dots) using electron beam lithography and lift-off tech-

\footnotetext{
${ }^{a)}$ Author to whom correspondence should be addressed. Electronic mail: maria_p_gonzalez_moral@yahoo.es
}

nique has been described in detail in Ref 4. $e$-beam lithography is used to define the desired structure into an electron sensitive resist (PMMA) which is coated on top of a $\mathrm{Si}(100)$ substrate. After lithography, $\mathrm{Ni}$ is deposited using magnetron sputtering. A final lift-off step removes the $\mathrm{Nb}$ on the PMMA. For the samples discussed in this article, magnetic dots have a diameter and thickness of 250 and $40 \mathrm{~nm}$, respectively. The size of the array is $190 \times 180 \mu \mathrm{m}$. The arrays have a rectangular symmetry with lattice parameters: $a \times b=600 \times 400 \mathrm{~nm}$ (see the inset of Fig. 1). Once the magnetic nanostructures are created on top of the substrate, they are covered with a superconducting $100 \mathrm{~nm}$ thick $\mathrm{Nb}$ film deposited via magnetron sputtering.

For transport measurements, a stripline is defined by standard optical lithography and ion beam etching. The pat-

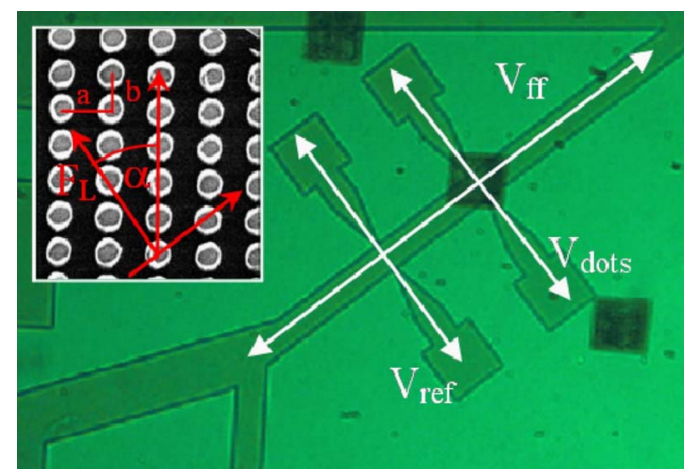

FIG. 1. (Color online) SEM image of the experimental arrangement of a $\mathrm{Nb}$ stripline (100 $\mu \mathrm{m}$ wide) with an array of $\mathrm{Ni}$ dots (see the dark area on the strip line and the enlarged inset) for the resistive measurement of the longitudinal voltage and the two pairs of Hall contacts for simultaneous determination of the Hall signal at the dot array (at the top) and at a reference point without magnetic dots (bottom pair of Hall contacts). The periodicity of the array is $a=600 \mathrm{~nm}$ and $b=400 \mathrm{~nm}$; the smaller lattice parameter $b$ forms an angle $\alpha$ with the direction of the Lorentz force (see inset). 


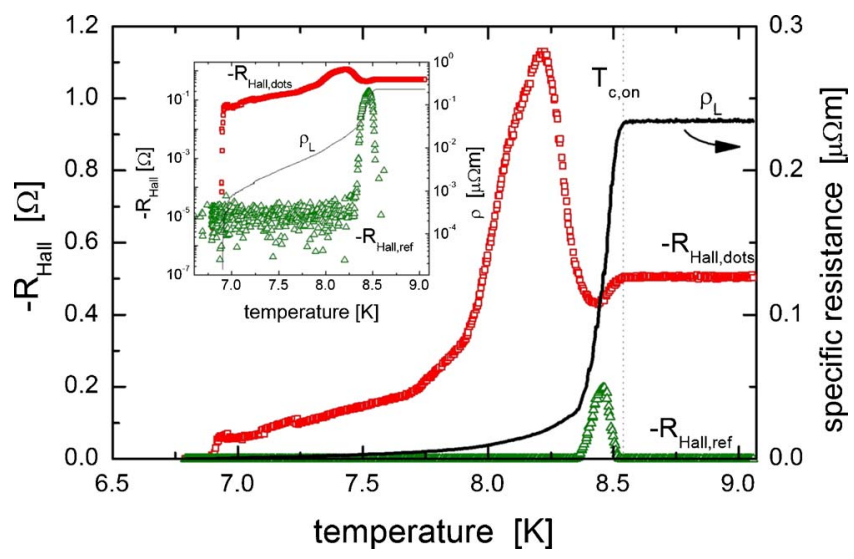

FIG. 2. (Color online) Temperature dependence of the longitudinal specific resistance $\rho_{L}$ (solid line) and inverted Hall signals $\left(-R_{\text {Hall }}\right)$ with (open squares) and without (open triangles) array of magnetic dots. It should be noted that $\left(-R_{\text {Hall, ref }}\right)$ is negative for $T>T_{c, \text { on }}$.

tern consists of a $100 \mu \mathrm{m}$ wide bridge placed under an angle with respect to the array of magnetic dots. The resulting angle $\alpha \sim 40^{\circ}$ (see the inset of Fig. 1) is defined by the directions of the Lorentz force $F_{L}$ (perpendicular to the direction of the applied current) with respect to the orientation of the rows of dots (smaller lattice parameter $b$ of the array). In previous works ${ }^{12}$ it has been shown that the maximum value of the Hall signal is obtained for guided motion under an angle of $30-40^{\circ}$ Furthermore, two pairs of Hall contact are added at positions including and excluding the array of magnetic dots, respectively.

The resulting arrangement allows simultaneous measurements of (i) the total longitudinal voltage $V_{L}$; (ii) the transversal (Hall) voltage $V_{\text {Hall,dots }}$ at the array of magnetic dots, and (iii) the transversal (Hall) voltage $V_{\text {Hall,ref }}$ at a reference position without magnetic dots as function of temperature, applied magnetic field, and applied current.

Thus, in the superconducting state a simultaneous measurement of the total amount of longitudinal flux transport ( $V_{L}$ represents the sum of the longitudinal voltage at the areas of the sample with dots and without dots, respectively) and the local transversal component of flux transport with and without magnetic dots is possible via this design. In the normal state, the normal resistance and the Hall resistance with and without magnetic dots are recorded.

\section{RESULTS AND DISCUSSION}

Figure 2 displays a typical set of measurements of the resistive transition and the corresponding behavior of the Hall signals with and without magnetic dots. For better visibility, the inverted Hall signals $-R_{\text {Hall }}$ are plotted. The normal state resistance of $R_{L}=46.9 \Omega$ corresponds to a specific resistance of $\rho_{L}=2.3 \times 10^{-7} \Omega \mathrm{m}$ that is slightly larger than the literature value of the specific resistance of $\mathrm{Nb}$ of 1.5 $\times 10^{-7} \Omega \mathrm{m}$ (Ref. 5) and could be an indication of the impact of the magnetic dots. The data obtained at the transition to the superconducting state provide an indication of the different mechanisms of vortex motion in the different areas of the sample.
First, for the longitudinal specific resistance $\rho_{L}$ an initial abrupt drop to about $15 \%$ of the normal state resistance indicates the onset of the transition at $T_{c, \text { on }} \cong 8.54 \mathrm{~K}$. It is followed by a broad tail that persists down to about $6.9 \mathrm{~K}$, where a second sharp drop of the resistance is measured (see logarithmic plot of the data in the inset of Fig. 2).

Second, for temperatures $T>T_{c, \text { on }}$ a small Hall resistance $R_{H, \text { ref }} \cong 0.04 \Omega$ is measured at the reference position (without magnetic dots). Using the standard expression $\Delta R_{H}=\left|R_{H}(-B)-R_{H}(B)\right|=2 B / n q d$, the charge carrier density $n$ can be derived from the Hall signal in the normal state. Here, $q$ and $d$ represent the electronic charge and the thickness of the film, respectively.

Inserting the experimental value $\Delta R_{H, \mathrm{ref}}(0.517 T)$ $=0.53 \mathrm{~m} \Omega$ yields a density of charge carriers of $n \approx 1.21$ $\times 10^{29} \mathrm{~m}^{-3}$, which is in good agreement with literature values. ${ }^{6,7}$

In contrast to the longitudinal resistance, $-R_{H, \text { ref }}$ shows a pronounced maximum at $T_{c}$. This maximum, together with the sign inversion $\left(-R_{H \text {,ref }}\right.$ is negative for $\left.T>T_{c, \text { on }}\right)$, is a clear indication for the existence of the anomalous Hall effect (AHE), which is common to superconducting systems. ${ }^{8-10}$ The AHE is caused by vortex motion with a directional component antiparallel to the current direction. ${ }^{11}$ Close to $T_{c}$ and in the superconducting regime, vortices are mobile. They are driven by the Lorentz force (perpendicular to applied current and magnetic field) and the Magnus force (antiparallel to the current). The Hall measurement records the component of motion along the current. With decreasing temperature, vortex pinning takes over and the Hall signal drops to zero at $T=8.33 \mathrm{~K}$. The result is a pronounced peak in a small temperature regime $(8.33-8.54 \mathrm{~K})$.

Finally, the temperature dependence of the Hall signal recorded at the magnetic dots is more complex. It seems to resemble the combination of both previously discussed dependencies. Above $T_{c, \text { on }}$ a relatively large negative Hall signal is observed for the magnetic dots. This could be caused by redistribution of the local normal current in the vicinity of the dots. Alteration of the applied magnetic field (only executed at room temperature) leads to a change of the signal $\Delta R_{H}=\left|R_{H}(-B)-R_{H}(B)\right|$ in agreement with the measurements of the reference, i.e., inversion of a magnetic field yields a change of the Hall signal $\Delta R_{\mathrm{Hall}, \mathrm{dot}} \approx 0.53 \mathrm{~m} \Omega$ (identical to the value obtained for the reference part, i.e., $\mathrm{Nb}$ without dots). From these data a similar density of charge carriers of $n \approx 1.21 \times 10^{29} / \mathrm{m}^{3}$ can be obtained. Thus, we conclude that the quality of the $\mathrm{Nb}$ is not seriously affected by the presence of the dots. The sight shift in $T_{c}$ has to be ascribed to the impact of the magnetic field of the dots upon the superconductor.

From the comparison of the field-dependent transition temperature in the reference part and the area of the dots, we can estimate that the local magnetic field due to the magnetic dots is about $6 \mathrm{mT}$.

Furthermore, at $T_{c \text {, on }}$ the Hall resistance initially drops due to the transition to the superconducting state. Then, a peak in the Hall resistance similar to but larger and more extended than the AHE of the reference signal occurs. This 

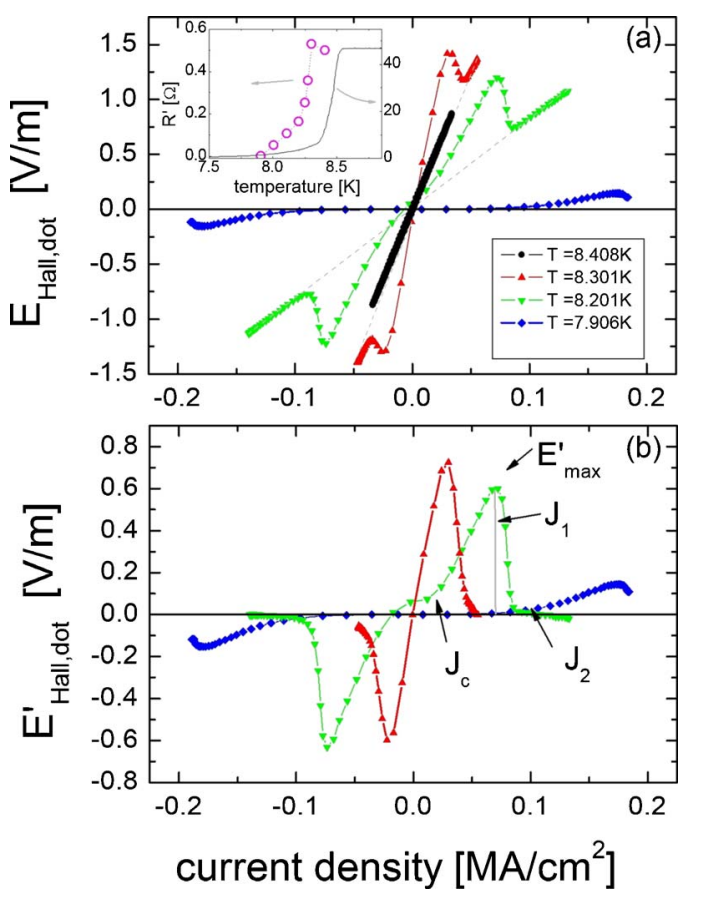

FIG. 3. (Color online) Set of VICs for different temperatures for the Hall measurement on the array of magnetic dots. The dashed lines in (a) represent the linear contribution $R_{\text {Hall,dot }}^{\prime}$ that is plotted as function of temperature in the inset together with the temperature dependence of longitudinal resistance $R_{L}$. In (b) the nonlinear contribution $V_{\text {Hall,dot }}^{\prime}$ is plotted for the VICs recorded in the superconducting state. Different current densities $\left(J_{c}, J_{1}, J_{2}\right)$ that characterize specific features of the VICs are marked for one of the VICs.

peak is followed by a broad tail that resembles the behavior of the longitudinal resistance $R_{L}$ (see the inset of Fig. 2).

A closer look at the voltage-current characteristic (VIC) of the Hall signal recorded at different temperatures for the sample area with dots reveals a complex behavior. Figure 3 shows a typical set of VICs obtained for different temperatures.

In contrast to the linear dependence measured for the normal state $T>T_{c}$, a nonlinear VIC is obtained for temperatures $T<T_{c}$. The nonlinear behavior can more easily be interpreted if the voltage signal is reduced by a linear contribution $R_{\text {Hall,dot }}^{\prime}$,

$$
V_{\text {Hall,dots }}^{\prime}=V_{\text {Hall,dots }}-R_{\text {Hall,dots }}^{\prime} I \text {, }
$$

with a linear contribution $R_{\text {Hall,dot }}^{\prime}$ being defined by the ohmic behavior observed for large currents [see the dashed lines in Fig. 3(a)]. The temperature dependence of this linear contribution shows a similar transition that is measured for the longitudinal resistance $R_{L}$ [see the inset of Fig. 3(a)]. It shows the same steepness but it is shifted to lower temperatures by about $0.22 \mathrm{~K}$. This shift can be explained by the reduction of the transition due to the local magnetic field of the magnetic dots. The nonlinear part of the VIC is shown in detail in Fig. 3(b). The first part of the VIC (low current densities) resembles the critical behavior. Up to a critical current density, $J_{c}$, no voltage is measured since the vortices are pinned. The critical current is zero at $T_{c}$ (see the VIC curve for $T=8.301 \mathrm{~K}$ ) and increases with decreasing temperature.

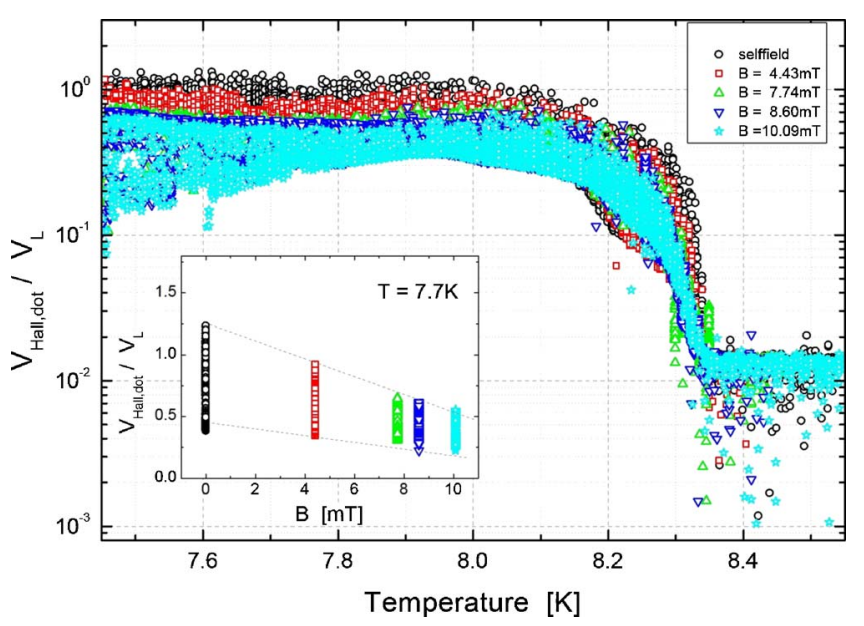

FIG. 4. (Color online) Ratio of the Hall signal at the magnetic dots and the longitudinal voltage as function of temperature for various applied magnetic fields $B$. The inset shows the field dependence of the ratio at $T=7.7 \mathrm{~K}$

Since the Hall contacts only record the contribution of vortex motion parallel and antiparallel to the direction of the applied current, the measured voltage is a clear indication for the manipulation of the direction of vortex motion for currents densities $J>J_{c}{ }^{11}$ Later in the article, we will show that the direction of vortex motion most likely coincides with the geometrical arrangement of the magnetic dots, i.e., we will demonstrate the presence of guided motion in this part of the phase diagram and regime of current densities.

An interesting feature is observed at larger current densities. The increase of the signal is reversed at $J_{1}$ [corresponding to $V_{\max }^{\prime}$; see Fig. 3(b)], i.e., the Hall voltage decreases and drops to zero. This behavior can only be explained by a redirection of the vortex motion. The vortices are exposed to the Lorentz force $\boldsymbol{F}_{L}=\left(\boldsymbol{J}-\boldsymbol{J}_{c}\right) \times \boldsymbol{B}$ with increases linear with applied current density. Up to current densities $J_{1}$, the vortices seem to be guided by the magnetic dots that are arranged under an angle with respect to $\boldsymbol{F}_{L}$. This leads to an increase of the Hall signal $V_{\text {Hall,dots }} \propto\left(J-J_{c}\right)$. If the driving force becomes too large, i.e., $J>J_{1}$, the vortices start to "ignore" the magnetic dots and flow in the direction of the Lorentz force. As a result the Hall signal reduces to zero. Similar effects have been observed for dimensional crossover of flux disorder where a 3D distorted vortex lattice reconverts to a $2 \mathrm{D}$ disordered vortex lattice and vice versa depending on the applied driving force. ${ }^{12}$ Finally, for current densities larger than $J_{2}$ vortex guidance is absent and the Hall signal $E_{\text {Hall,dot }}^{\prime}$ vanishes completely.

The degree of guidance (i.e., relative amount of vortices that is guided by the rows of magnetic dots) of vortices by the rows of magnetic dots is quantified by evaluating the ratio of the two voltages $V_{\text {Hall,dot }} / V_{L}$ in the different current regimes. Figure 4 shows a typical set of data obtained for one of the samples.

In order to select the state of moving vortices and to avoid zero division, only data for $\left|V_{L}\right|>1 \mathrm{mV}$ are shown. In the normal state a ratio of $V_{\mathrm{Hall} \text {,dot }} / V_{L} \approx 0.01$ is measured for all applied magnetic fields. A relatively large Hall signal of the dot area was already visible in Fig. 2. This signal (and, consequently, the nonzero ratio) is too large to be explained 
by an insufficient patterning, e.g., a misalignment of the Hall contacts. It probably has to be ascribed to the modification of the local current distribution due to the array of magnetic dots in this part of the sample.

In the superconducting regime $\Gamma_{\text {guidance }}=V_{\text {Hall, dot }} / V_{L}$ characterizes the guidance of vortices. $\Gamma_{\text {guidance }}$ represents the ratio of guided and total number of moving vortices. Here, $\Gamma_{\text {guidance }}=1$ means complete guidance, whereas $\Gamma_{\text {guidance }}=0$ stands for no guidance. Within a small temperature regime below $T_{c}, \Gamma_{\text {guidance }}$ increases from 0.01 (minimum value due to redirection of the normal state current) to about unity, i.e., $\Gamma_{\text {guidance }} \approx 1$. The maximum value is obtained at the maxima of the Hall signal $E_{\max }^{\prime}$ (see Fig. 3 ). Thus, $\Gamma_{\text {guidance }} \approx 1$ means that nearly all vortices that move in the superconducting sample move along the lines of magnetic dots. Thus, we observed a complete guidance of vortices for $J=J_{1}$, or vortices are only moving along the rows of magnetic dots. For currents larger or smaller than $J_{1}$ the guidance is less effective. However, even in these cases the guidance is still quite effective. As indicated in the inset of the figure, more than $40 \%$ of the vortices is still guided by the dots for $T$ $\approx 7.7 \mathrm{~K}$ and self-field $\left(B_{\text {applied }}=0\right)$ for $J \neq J_{1}$. With increasing magnetic field, the guidance becomes slightly less effective (see the inset of Fig. 4). This might be an indication for the additional motion of vortices between the rows of magnetic dots (interstitial vortices). These vortices are not directly affected by the rows of dots; therefore, their guidance will be less effective.

It should be noted that, in contrast to other experiments on patterned conventional superconductors, the effect of guidance could be demonstrated over quite a broad temperature regime $(\sim 1 \mathrm{~K})$. At lower temperatures the driving currents become too large and heating at contacts restricts the measurement.

\section{CONCLUSIONS}

In summary, we have analyzed and quantified the effect of guidance of vortices by magnetic dots using simultaneous resistive measurements of the longitudinal and Hall voltage due to vortex motion. We could demonstrate that effective guidance of vortices is present only in a limited regime of driving force that is characterized by a current density, $J_{c}$ $<J<J_{2}$. If the vortices are exposed to a larger driving force $\left(f_{p}>\phi_{o} J_{2}\right)$ they start to be redirected and move in the direction of the Lorentz force. In the optimal case $\left(J \approx J_{1}\right)$ all vortices are guided by the magnetic dots, i.e., $\Gamma_{\text {guidance }} \approx 1$. For diverging current densities $\left(J \neq J_{1}\right)$ the amount of guided vortices decreases. Nevertheless, as long as vortex motion is detected (i.e., for $|V|>1 \mathrm{mV}$ ), most of the vortices seem to be guided by the dots (typically we observed $\Gamma_{\text {guidance }}>0.4$ for zero-applied field). Moreover, the effect of guidance is reduced with increasing magnetic fields. The latter might be an indication for the reduced guidance of interstitial vortices that are formed at larger applied magnetic fields.

Generally, vortex manipulation is an interesting aspect for basic research and potential applications of superconductivity in fluxonic devices. The understanding and quantifications of guidance that is demonstrated in this work can be of importance for these activities.

\section{ACKNOWLEDGMENTS}

The authors would like to thank J. V. Anguita, E. M. González, J. L. Vicent, I. K. Schuller, R. Kutzner, N. Klein, and A. Offenhäuser for their valuable support. M.P.G. wants to thank the Spanish Ministerio de Educación y Ciencia for the fellowship BES-2003-0824.

\footnotetext{
${ }^{1}$ M. Andersson, J. C. Cuevas, and M. Fogelström, Physica C 367, 117 (2002).

${ }^{2}$ M. Lange, M. J. Van Bael, Y. Bruynseraede, and V. V. Moshchalkov, Phys. Rev. Lett. 90, 197006 (2003).

${ }^{3}$ J. I. Martín, M. Vélez, J. Nogués, and I. K. Schuller, Phys. Rev. Lett. 79, 1929 (1997).

${ }^{4}$ J. I. Martín, M. Vélez, R. Morales, J. M. Alameda, J. V. Anguita, F. Briones, and J. L. Vicent, J. Magn. Magn. Mater. 249, 156 (2002).

${ }^{5}$ A. M. James and M. P. Lord, Macmillan's Chemical and Physical Data (Macmillan, London, UK, 1992).

${ }^{6}$ T. P. Beaulac, Phys. Rev. B 23, 3617 (1981).

${ }^{7}$ C. M. Hurd, The Hall Effect in Metals and Alloys (Plenum, New York, 1972).

${ }^{8}$ S. J. Hagen, C. J. Lobb, R. L. Greene, M. G. Forrester, and J. H. Kang, Phys. Rev. B 41, 11630 (1990).

${ }^{9}$ S. J. Hagen, A. W. Smith, M. Rajeswari, J. L. Peng, Z. Y. Li, R. L. Greene, S. N. Mao, X. X. Xi, S. Bhattacharya, Q. Li, and C. J. Lobb, Phys. Rev. B 47, 1064 (1993).

${ }^{10}$ H. Van Beelen, J. P. Van Braam Houckgeest, H. M. Thomas, C. Stolk, and R. De Bruyn Ouboter, Physica 36, 241 (1967).

${ }^{11}$ R. Wördenweber, J. S. K. Sankarraj, P. Dymashevski, and E. Hollmann, Physica C 434, 101 (2006).

${ }^{12}$ R. Wördenweber, P. Dymashevski, and V. R. Misko, Phys. Rev. B 69,
} 184504 (2004). 